\title{
DIFERENÇAS DE PERCEPÇÃO DE ADOTANTES E NÃO-ADOTANTES QUANTO AO USO DE SERVIÇOS DE MOBILE BANKING E SUA RELAÇÃO COM AS CARACTERÍSTICAS INDIVIDUAIS DE INOVATIVIDADE
}

\section{DIFFERENCES IN PERCEPTION OF NON-ADOPTERS AND ADOPTERS FOR THE USE OF SERVICES FOR MOBILE BANKING AND IT'S RELATIONSHIPWITH THE CHARACTERISTICS OF INDIVIDUAL INNOVATIVENESS}

\author{
Anatália Saraiva Martins Ramos \\ Universidade Federal do Rio Grande do Norte \\ Iris Linhares Pimenta \\ Universidade Federal do Rio Grande do Norte \\ Paula Augusta Barbosa Rodrigues \\ Universidade para o Desenvolvimento da Região do Pantanal
}

\section{RESUMO}

O presente trabalho buscou analisar as percepções dos consumidores quanto à adoção de novas tecnologias de serviços bancários baseados na mobilidade - o Mobile Banking. Tendo como base a clássica Teoria de Difusão de Inovações (TDI) proposta por Rogers (1995), foi empreendida uma investigação quantitativa do tipo exploratória com 96 adotantes ou potenciais adotantes do mobile banking escolhidos de forma não-probabilística por conveniência. A análise estatística ocorreu em três etapas: análise descritiva, teste de variância e correlação. Constatou-se que os não adotantes têm uma visão mais negativa dos atributos da inovação (vantagem relativa, observabilidade, compatibilidade e complexibilidade). Confirmou-se que as características típicas de inovatividade influenciam a percepção acerca dos serviços de Mobile Banking na maior parte das variáveis. 0 artigo finaliza com as implicações teóricas e gerenciais decorrentes dos resultados.

Palavras-chave: Adoção de inovação. Teoria da difusão de inovações. Mobile banking. Inovatividade

\begin{abstract}
This study investigates the perceptions of consumers regarding the adoption of new technologies based on mobile banking - Mobile Banking. Based on the classical theory of Diffusion of Innovations (TDI) proposed by Rogers (1995), was undertaken a quantitative investigation of the exploratory type with 96 adopters or potential adopters of mobile banking chosen in a non-random. Statistical analysis occurred in three stages: descriptive analysis of variance and correlation. It was found that nonadopters have a more negative view of the attributes of innovation (relative advantage, observability, compatibility and complexity). It was confirmed that the typical characteristics of innovativeness influence the perception of Mobile Banking Services in most of the variables. The article concludes with theoretical and managerial implications from the results.
\end{abstract}

Keywords: Adoption of innovation. Diffusion of innovations theory. Mobile banking. Innovativeness 


\section{INTRODUÇÃO}

No Brasil, o número de telefones móveis já ultrapassa o número de clientes da telefonia fixa. Em 2005, havia 59,3\% de moradias com telefonia móvel contra 48,1\% com telefonia fixa (IBGE, 2005). Esse panorama é reflexo da revolução tecnológica da mobilidade, capaz de atingir o cotidiano das pessoas, modificando suas rotinas e formas de tomar decisões.

Os avanços tecnológicos da mobilidade trouxeram também uma outra inovação, a partir da convergência com o acesso a Internet, através da Wireless Aplication (WAP). A principal vantagem do acesso à Internet pela telefonia móvel é o acesso totalmente portátil em tempo real aos mesmos recursos e ferramentas de informação que, até recentemente, somente eram acessíveis pelo computador, conforme Kalakota e Robinson (2002, p. 17).

Este estudo busca descrever a percepção do consumidor de produtos inovadores associada com seu perfil de adoção/difusão do Mobile Banking. Existem vários modelos teóricos que buscam investigar essa percepção como precursora da adoção. Conforme Hernandez e Mazzon (2006, p. 2), uma das linhas de pesquisa mais importantes em adoção de tecnologias refere-se aos determinantes de aceitação individual, fundamentados na Teoria da Ação Racional (TRA) e na Teoria do Comportamento Planejado (TPB), que por sua vez geraram os pressupostos do Modelo de Aceitação de Tecnologia (TAM). Uma segunda linha tem investigado essa adoção a partir das características da nova tecnologia, sob a perspectiva da Teoria da Difusão de Inovação.

A pesquisa proposta foca-se nessa segunda linha teórica, utilizando a Teoria da Difusão de Inovações (TDI), para buscar conhecer a percepção que os consumidores têm acerca do uso de serviços de Mobile Banking. Esta escolha pela TDI se deve a sua integração metodológica, por ser a abordagem que explica o processo de adoção de novas tecnologias. Sendo assim, entende-se que a TDI poderá responder às questões de pesquisa com mais propriedade, poder explicativo e relevância acadêmica tão bem quanto os demais modelos encontrados na literatura.

Especificamente, o presente estudo teve como objetivo descrever o perfil dos "adotantes" do Mobile Banking; identificar as diferentes percepções dos "adotantes" e "não adotantes" de serviços de Mobile Banking com relação às variáveis: vantagem relativa, compatibilidade, observabilidade e complexidade; e investigar se há correlação entre a percepção dos serviços de Mobile Banking e as características individuais presentes nas categorias de adotantes proposto por Rogers (1995).

\section{REFERENCIAL TEÓRICO}

A Internet é um sistema mundial de redes de computadores, interligados pelo Protocolo de Internet, que possibilita o acesso a informações armazenadas em bancos de dados do mundo inteiro (Turban; Mclean; Wetherbe, 2004, p. 124). Apesar das diversas formas de acesso e serviços disponibilizados, a rede ainda esta em desenvolvimento e seus avanços vem se refletindo em todo o mundo, embora com intensidade diferenciada em função do nível de desenvolvimento das sociedades ou de outros fatores como políticos e culturais. Conforme dados de pesquisa realizada em 2007 pelo Nielsen//NetRatings, $16,9 \%$ da população mundial utiliza-se da rede, ou seja, em torno de 1,1 bilhão de pessoas encontramse conectadas.

Na esteira do desenvolvimento tecnológico, foi adotado o WAP (Wireless Application Protocol), que é um padrão internacional para aplicações que utilizam comunicações sem fio, buscando a convergência de duas tecnologias de rede: a transmissão de dados sem fio e a Internet. A ideia básica é utilizar os conceitos da Internet modificando-os para que eles possam ser úteis dentro das limitações de uma rede sem fio e de aparelhos com recursos restritos. A navegação é feita por meio de um mini-browser (navegador) que traz informações para a tela do aparelho móvel em tempo real (Hung; Ku; Chang, 2002, p.3). 


\section{MOBILE BANKING}

O setor bancário continua sendo considerado como um dos que mais investe em tecnologia de informação. Segundo Hernandez e Mazzon (2006, p.5), foi a partir dos anos 80 e início dos anos 90 que a literatura sobre adoção de tecnologias bancárias desenvolveu-se, principalmente após o surgimento daquelas que possibilitaram o acesso remoto aos serviços bancários como: cartões magnéticos, caixas automáticos, Internet Banking e Mobile Banking.

A Internet foi o alvo dos serviços bancários de auto-atendimento, criando toda uma gama de aplicações denominada de Internet Banking (IB). Em seguida, nunca escala de evolução tecnológica, surgiu o Mobile Banking (MB), a partir da inclusão de uma chave criptográfica do banco dentro do próprio chip nos celulares GSM.

No Brasil, com a vantagem da capilaridade das redes de telecomunicações móveis, existem perto de 150 milhões de assinantes, sendo 122,7 milhões pré-pagos (81,47\%) e 27,9 milhões pós-pagos (18,53\%), segundo a Agência Nacional de Telecomunicações (Anatel, 2008). Com um mercado com este porte, os bancos começaram a visualizar uma oportunidade de negócios. 0 pioneiro na disponibilização do serviço foi o Banco do Brasil, que oferece por 2,50 reais mensais (cerca de US\$ 1.40) a possibilidade de seus correntistas cadastrados realizarem consultas de extratos e saldos, efetuarem transferências eletrônica de fundos, pagamento de títulos e boletos e receberem alertas via SMS (Short Message Service) sobre sua movimentação bancária pelo telefone móvel. No ano em que esta inovação chegou ao mercado, 240 mil clientes adotaram a solução de Mobile Banking, totalizando 1,3 milhão de transações no período. Desde o lançamento, o crescimento médio do serviço atingiu índices mensais entre 8\% e 10\% (Oliveira, 2006).

\section{TEORIA DA DIFUSÃO DA INOVAÇÃO}

0 modelo da Difusão de Inovações (Diffusion of Innovations Theory) proposto por Roger em 1962 é um dos mais referendados na literatura e tem sido utilizado desde os anos 60 para explicar o processo de adoção de novas tecnologias (Hernandez, Mazzon, 2006, p. 2).

Rogers (1995, p. 10) define difusão como: "o processo pelo qual uma inovação é comunicada, através de um canal, entre os membros de um sistema social". As características ou atributos de uma inovação que podem influenciar na taxa de adoção foram definidas como: - Vantagem relativa: pode ser definida como o grau em que uma inovação é percebida como superior às alternativas existentes ou melhor do que a ideia que está sendo substituída. 0 grau de vantagem relativa pode ser medido em função da rentabilidade econômica, prestígio social, baixo custo inicial, economia de tempo e esforço, maior conforto e recompensa imediata.

- Compatibilidade: corresponde ao grau em que uma inovação é percebida como consistente com os valores, experiências passadas e necessidades dos potenciais adotantes. Neste último caso, as pessoas responsáveis pela difusão (agentes de mudança), procuram determinar essas necessidades, e em seguida atendê-las por intermédio da recomendação de inovações. Para ser compatível, a inovação deve também se aproximar da maneira mais similar a já utilizada pelos usuários.

- Complexidade: refere-se ao grau em que uma inovação é percebida como difícil de ser compreendida e utilizada. Infere-se que quanto maior a percepção de facilidade no uso, maior será a taxa de adoção.

- Observabilidade: é o grau em que os benefícios ou atributos da inovação podem ser observados, imaginados ou descritos aos potenciais adotantes.

- Experimentabilidade: corresponde ao grau em que uma inovação pode ser testada antes da sua adoção efetiva. 
Rogers (1995, p.32) afirmou que uma variação de 49\% a 87\% na taxa de adoção podem ser explicadas pela percepção que o potencial adotante tem dessas cinco características.

As pesquisas com base na Teoria da Difusão de Inovação foram adicionando novas características (ou construtos), além dessas cinco originais. Pesquisas como a de Moore e Benbasat (1991), adicionaram os construtos Imagem e Voluntariado na Utilização. Esse primeiro diz respeito à percepção do indivíduo com relação à utilização de uma inovação e a melhoria da sua imagem ou status, enquanto que o Voluntariado na Utilização foi definido como o grau no qual o uso de uma inovação é percebido como sendo voluntário ou de livre escolha.

Uma variável importante que pode afetar a taxa de adoção de uma inovação foi definida por Yi, et al. (2006) como "personalidade inovadora", ou seja, as diferentes reações que os possíveis adotantes têm diante de uma nova tecnologia. A partir desse conceito, é possível classificar os indivíduos ou grupos em categorias, de acordo com sua maior ou menor predisposição para adotar novas ideias.

Rogers (1995) identificou cinco categorias de adoção. Os inovadores, os primeiros a adotarem uma inovação; os primeiros adotantes, que são influenciadores e líderes; a maioria inicial adotante, aqueles que adotam antes da média; a maioria tardia, composta de pessoas céticas as quais só adotam a ideia após metade de adotantes do sistema social já terem adotado e os retardatários, também conhecidos como tradicionais, são os últimos a adotarem

determinada ideia.

Midgley e Dowling (1978 apud Rodrigues e Marchetti, 2008) propõem que, para definir uma pessoa como inovadora nata, deve-se utilizar dois critérios: o grau de receptividade a novas idéias e o grau com que o processo de decisão de compra do consumidor depende da influência de outros membros do sistema social. Desta maneira, um consumidor pode ser um adotante inicial de uma inovação sem necessariamente ser um inovador nato, pois há uma série de variáveis intervenientes atuando no processo de adoção, como o interesse na categoria de produto e efeitos situacionais como recursos financeiros.

Semelhante ao estudo realizado por Rogers (1995), e baseados no conceito de que existem indivíduos mais propensos a inovar que outros, Parasuraman e Colb (2002, p. 53) realizaram pesquisa a qual obtiveram resultado semelhante. Os autores concluíram que as primeiras pessoas a chegar são "exploradores", altamente motivadas e sem medo. As seguintes são "pioneiras", que desejam os benefícios da nova terra, mas são mais práticas a respeito das dificuldades e dos perigos. 0 terceiro grupo é composto por dois tipos: os "céticos", que precisam ser convencidos dos benefícios dessa nova fronteira, e os "paranóicos", que estão convencidos dos benefícios, mas são extraordinariamente preocupados com os riscos de se viver neste novo conceito. No último grupo, estão os "retardatários", podem nunca vir a adotar produtos inovadores, a menos que sejam forçados a isso.

Estudos sobre adoção de inovação devem considerar os traços pessoais, tais como o perfil de 'inovatividade' individual, tendo em vista que são determinantes relevantes para a adoção e podem ser mais importante ainda nas decisões de adotantes potenciais (Lu, Yao e Yu, 2005).

\section{METODOLOGIA}

A pesquisa é caracterizada como exploratória, do tipo levantamento de dados (survey). A primeira etapa da pesquisa constituiu-se da obtenção de dados secundários através de pesquisa bibliográfica. A segunda etapa caracterizou-se pela coleta de dados diretamente na fonte através da aplicação de questionário estruturado (Anexo 1). Antes da pesquisa de campo propriamente dita, foi feita a adequação do instrumento de coleta de dados (questionário), a partir da aplicação de um pré-teste com pessoas, usuárias ou não de serviços de Mobile Banking no telefone móvel.

Foram aplicados 98 questionários entre estudantes do curso de Graduação em Administração da UFRN no período de 17 à 28 de abril de 2008, através de uma amostra não-probabilística por 
conveniência. 96 questionários foram considerados válidos para a análise.

Para responder ao questionário, era necessário possuir telefone móvel com a tecnologia WAP e possuir conta bancária.

A primeira parte do instrumento de pesquisa é referente às questões sobre uso de serviços bancários e de Internet no telefone móvel. Posteriormente, eram indagados sobre questões voltadas para a percepção do serviço de Mobile Banking. A terceira parte do questionário possibilitava o levantamento do perfil de inovatividade do respondente, baseado nas variáveis da Teoria da Difusão de Inovação de Rogers (1995). Para medir essas variáveis foi utilizado o escalonamento do tipo Likert.

O tratamento estatístico foi realizado com o auxílio do Statistical Package for the Social Sciences (SPSS), versão 15, contemplando duas análises estatísticas: o teste de análise de variância (ANOVA) e a correlação de Spearman. $O$ teste de análise de variância foi realizado a fim de identificar a percepção, segundo os atributos de uma inovação, dos "adotantes" e "não adotantes" acerca do Mobile Banking. A análise de correlação teve como objetivo testar as relações existentes entre as percepções de "adotantes" e "não adotantes", quanto aos serviços de Mobile Banking e as características individuais que compõem as cinco categorias de adotantes descritas por Rogers (1995). Para esta pesquisa, optouse por adotar o nível de significância de $10 \%$ por seu caráter exploratório.

\section{RESULTADOS}

Com relação ao perfil dos adotantes de serviços de Mobile Banking (MB), 52,2\% são do sexo masculino e 47,8\% do sexo feminino. Em termos de gênero, o teste de chi quadrado mostrou que não há diferença estatística em termos de adoção do MB, o que mostra uma disseminação igualitária entre os sexos. A maioria dos respondentes é solteira (78,26\%). A maior parte concentra-se na faixa etária que vai dos 20 aos 30 anos $(82,61 \%)$ e com renda familiar que vai de seis a dez salários mínimos (56,53\%), que corresponde a cerca de US\$22.000 a US\$ 37.000 anuais. Ressalte-se que a coleta dos dados foi feita junto a universitários, o que é coerente com esse perfil.

A grande maioria dos respondentes $(85,4 \%)$ é constituída de correntistas de bancos estatais, sendo 61 estudantes com conta corrente no Banco do Brasil, primeiro banco a oferecer o serviço, seguidos de 21 da Caixa Econômica Federal. Conforme a Tabela 1, cruzando as variáveis Banco e Adoção de MB, observa-se que apenas 23 universitários são utilizadores dos serviços bancários pelo aparelho móvel, aproximadamente $1 / 4$ da amostra pesquisada. No entanto, os dados da pesquisa mostraram que ainda há um percentual de $22 \%$ da amostra que nunca ouviram falar em mobile banking (MB).

\begin{tabular}{|c|c|c|c|}
\hline $\begin{array}{c}\text { Tabela 1- } \\
\text { Distribuição dos } \\
\text { respondentes por } \\
\text { Bancos de acordo } \\
\text { com a adoção do MB } \\
\text { Bancos }\end{array}$ & $\begin{array}{c}\text { Usuário de } \\
\text { algum serviço } \\
\text { de MB }\end{array}$ & $\begin{array}{c}\text { Nunca utilizou } \\
\text { MB }\end{array}$ & $\begin{array}{c}\text { Percentual } \\
\text { Total }\end{array}$ \\
\hline Banco do Brasil & 15 & 46 & 61 \\
\hline Caixa Econômica & 4 & 17 & 21 \\
\hline Bradesco & 3 & 5 & 8 \\
\hline HSBC & $\mathrm{O}$ & 1 & 1 \\
\hline Itaú & $\mathrm{O}$ & 1 & 1 \\
\hline Real & 1 & 3 & 4 \\
\hline Total & 23 & 73 & 96 \\
\hline
\end{tabular}

Fonte: Autores

A tabela 2 apresenta os resultados do cruzamento das variáveis 'Frequência de uso do Internet Banking' com 'adoção do MB'. 0 teste de Tukey B mostra que as maiores médias (onde 1 é 'não usuário de MB' e 2 é 'usuário de MB') estão entre os que são mais assíduos no uso do Internet banking. 
Diferenças de percepção de adotantes e não-adotantes quanto ao uso de serviços de mobile banking e sua relação com as características individuais de inovatividade

\begin{tabular}{l|c|c}
\hline $\begin{array}{c}\text { Tabela 2-Usuário de MB e } \\
\text { sua relação com a frequiência } \\
\text { de uso do IB Freqüêcia de } \\
\text { Uso do Internet Banking }\end{array}$ & Respondentes (N) & $\begin{array}{c}\text { Média da variável } \\
\text { Adoção do MB } \\
\text { alpha }=\mathbf{0 , 1 0}\end{array}$ \\
\hline \hline Nunca usei & 20 & 1,35 \\
\hline Mensalmente & 23 & 1,61 \\
\hline Semanalmente & 48 & 1,63 \\
\hline Todos os dias & 5 & 1,80 \\
\hline Total & 23 & 73 \\
\hline
\end{tabular}

Fonte: Autores

Os dados corroboram com Saccol et al. (2005, p.50) que afirmam: "a experiência de um adotante com determinada tecnologia poderá influenciar sua percepção quanto a uma inovação que surja na seqüência, especialmente se elas dizem respeito a tecnologias ou ideias semelhantes". No teste de chi quadrado, ficou evidenciado que os que usam o Internet banking estão mais propensos a serem utilizadores de mobile banking (chi=6,283, nível de significância=0,09).

Com relação à utilização serviços pelo telefone móvel celular, constatou-se que uma pequena parte dos utilizadores de telefone móvel baixa jogos $(13,5 \%)$, músicas $(29,2 \%)$, acessa e-mails $(12,5 \%)$ e utilizase de mensageiros eletrônicos - chats $(9,4 \%)$.

Com relação às variáveis 'facilidade de operação', 'versatilidade', 'segurança' e 'facilidade em contar aos outros', pode-se observar na Tabela 3 que não há diferenças estatísticas significativas entre as médias de percepções dos "adotantes" e "não adotantes" acerca dos serviços de Mobile Banking.

Tabela 3: Percepção dos consumidores quanto aos atributos do Mobile Banking em relação aos adotantes do MB.

\begin{tabular}{l|c|c|c|c|c|c|c}
\hline $\begin{array}{c}\text { ATRIBU } \\
\text {-TOS DO } \\
\text { SERVIC } \\
\text { O DE } \\
\text { MB+ }\end{array}$ & $\begin{array}{c}\text { Serviços } \\
\text { são } \\
\text { caros* }\end{array}$ & $\begin{array}{c}\text { Trans- } \\
\text { missão é } \\
\text { lenta* }\end{array}$ & $\begin{array}{c}\text { Operaçáo } \\
\text { é fácil }\end{array}$ & $\begin{array}{c}\text { Nåo é } \\
\text { versátil }\end{array}$ & $\begin{array}{c}\text { Tem } \\
\text { menos } \\
\text { segurança }\end{array}$ & $\begin{array}{c}\text { Elimina } \\
\text { barreiras } \\
*\end{array}$ & $\begin{array}{c}\text { Facilidade } \\
\text { em contar } \\
\text { aos outros }\end{array}$ \\
\hline $\begin{array}{c}\text { Adotante } \\
\text { s de MB }\end{array}$ & 2,70 & 3,17 & 3,96 & 2,48 & 3,13 & 3,78 & 4,13 \\
\hline \hline $\begin{array}{l}\text { Não- } \\
\text { adotantes } \\
\text { de MB }\end{array}$ & 3,07 & 2,78 & 3,81 & 2,58 & 3,38 & 4,16 & 3,79 \\
\hline $\begin{array}{l}\text { Média } \\
\text { geral++ }\end{array}$ & 2,98 & 2,88 & 3,84 & 2,55 & 3,32 & 4,07 & 3,88 \\
\hline
\end{tabular}

+ A média da percepção dos atributos do MB é considerada a partir da escala 1- discordo totalmente a 4concordo totalmente

* Há diferença estatística entre os dois grupos, para nível de significância de 0,10

Fonte: Autores

Nas variáveis 'custo dos serviços', 'transmissão de dados' e 'eliminação de barreiras' observa-se que os "adotantes" percebem de forma diferente dos "não adotantes" quanto aos atributos de adoção do Mobile Banking. Os adotantes discordam mais que os não adotantes que o serviço móvel seja caro e que o MB elimine barreiras e concordam mais que a transmissão seja mais lenta.

A tabela 4 descreve a relação que há os dois grupos (adotantes e não adotantes de $\mathrm{MB}$ ) e as características do consumidor quanto a seu perfil de inovatividade. Em termos descritivos, os que são adotantes de MB são os que mais tendem a concordar com os aspectos positivos da adoção de produtos inovadores. 
Tabela 4: Perfil de inovatividade em relação aos adotantes do MB

\begin{tabular}{l|c|c|c|c|c|c}
\hline $\begin{array}{c}\text { PERFIL } \\
\text { DE } \\
\text { INOVATI } \\
\text {-VIDADE+ }\end{array}$ & $\begin{array}{c}\text { Necessito } \\
\text { da ajuda } \\
\text { dos outros }\end{array}$ & $\begin{array}{c}\text { Mais } \\
\text { controle } \\
\text { sobre as } \\
\text { vidas }\end{array}$ & $\begin{array}{c}\text { Pouca } \\
\text { segurança } \\
\text { em } \\
\text { operações } \\
\text { financeiras }\end{array}$ & $\begin{array}{c}\text { Sou um } \\
\text { dos } \\
\text { primeiros } \\
\text { a adotar } \\
\text { tecnologia } \\
*\end{array}$ & $\begin{array}{c}\text { Pouco } \\
\text { confiante }\end{array}$ & $\begin{array}{c}\text { Mais } \\
\text { eficiente } \\
\text { na } \\
\text { profissão* }\end{array}$ \\
\hline $\begin{array}{l}\text { Adotantes } \\
\text { de MB }\end{array}$ & 1,35 & 3,78 & 2,83 & 3,35 & 2,30 & 4,52 \\
\hline $\begin{array}{l}\text { Não- } \\
\text { adotantes } \\
\text { de MB }\end{array}$ & 1,58 & 3,97 & 3,07 & 2,55 & 2,68 & 4,21 \\
\hline $\begin{array}{l}\text { Média } \\
\text { geral }\end{array}$ & 1,52 & 3,93 & 3,01 & 2,74 & 2,59 & 4,28 \\
\hline
\end{tabular}

+ A média das variáveis do perfil de inovatividade é considerada a partir da escala 1- discordo totalmente a 4concordo totalmente

* Há diferença estatística entre os dois grupos, para nível de significância de 0,10

Fonte: Autores

\begin{tabular}{|c|c|c|c|c|c|c|}
\hline $\begin{array}{r}\text { Perfil de } \\
\text { inovativid } \\
\text { ade } \longrightarrow \\
\text { versus } \\
\text { Atributos } \\
\downarrow \\
\end{array}$ & $\begin{array}{c}\text { Necessito } \\
\text { de ajuda } \\
\text { dos outros }\end{array}$ & $\begin{array}{c}\text { Traz mais } \\
\text { controle } \\
\text { sobre as } \\
\text { vidas }\end{array}$ & $\begin{array}{c}\text { Pouca } \\
\text { segurança } \\
\text { em } \\
\text { operações } \\
\text { financeiras }\end{array}$ & $\begin{array}{c}\text { Sou um } \\
\text { dos } \\
\text { primeiros } \\
\text { a adotar } \\
\text { tecnologia }\end{array}$ & $\begin{array}{c}\text { Pouco } \\
\text { confiante }\end{array}$ & $\begin{array}{c}\text { Mais } \\
\text { eficiente } \\
\text { na } \\
\text { profissão }\end{array}$ \\
\hline $\begin{array}{l}\text { Serviços } \\
\text { são caros }\end{array}$ & 0,027 & $-0,004$ & 0,185 & 0,099 & 0,179 & 0,096 \\
\hline $\begin{array}{l}\text { Transmissã } \\
\text { oé lenta }\end{array}$ & 0,098 & $-0,183$ & 0,019 & 0,108 & 0,018 & $-0,125$ \\
\hline $\begin{array}{l}\text { Operação é } \\
\text { fácil }\end{array}$ & $-0,219(*)$ & $0,295(* *)$ & 0,022 & 0,199 & $-0,139$ & 0,308 (*) \\
\hline $\begin{array}{l}\text { Não é } \\
\text { versatile }\end{array}$ & 0,093 & $-0,125$ & $0,232(*)$ & $-0,042$ & $0,241(*)$ & $-0,070$ \\
\hline $\begin{array}{l}\text { Menos } \\
\text { segurança } \\
\text { que formas } \\
\text { tradicionai } \\
\mathrm{s}\end{array}$ & $0,248(*)$ & $-0,061$ & 0,373 (**) & $-0,057$ & 0,417 (**) & 0,034 \\
\hline $\begin{array}{l}\text { Elimina } \\
\text { barreiras }\end{array}$ & $-0,121$ & 0,475 (**) & 0,072 & $0,210(*)$ & $-0,058$ & 0,328 (**) \\
\hline $\begin{array}{l}\text { Facilidade } \\
\text { em contar } \\
\text { aos outros }\end{array}$ & $-0,178$ & 0,407 (*w) & 0,058 & 0,124 & $-0,129$ & $0,254(*)$ \\
\hline $\begin{array}{l}\text { Mais } \\
\text { difícil de } \\
\text { usar } \\
\text { comparati- } \\
\text { vamente }\end{array}$ & 0,243 (*) & $-0,171$ & 0,023 & $-0,117$ & 0,174 & $-0,115$ \\
\hline
\end{tabular}

** Correlação é significante ao nível de 0,01 .

Fonte: Autores

Em relação ao atributo do usuário que tem facilidade de operação do MB, identifica-se que, do ponto de vista da inovatividade, o respondente dá mais importância ao uso de ferramentas automatizadas para ganhar eficiência no seu trabalho, tem um pensamento positivo de que a tecnologia permite-o ter mais controle do que faz da sua vida sentem-se mais independente dos outros para lidar com tecnologia. Essas correlações mostram que o modelo de Rogers (1995) também foi corroborado para o ambiente de uso do mobile banking.

Os utilizadores que não atribuem o $\mathrm{MB}$ a condição de ferramenta versátil são os que são mais propensos a não terem confiança e segurança no uso de tecnologias, em geral. Na mesma direção, os respondentes que sentem mais dificuldade em utilizar os serviços de MB em comparação aos outros tipos de serviços bancários são os que tendem a necessitar mais de ajuda dos outros. 
No quadro de análise de correlação, identifica-se que as variáveis ligadas ao custo do serviço do MB e da percepção de lentidão de transmissão não tiveram correlação com o perfil de inovatividade.

\section{CONCLUSÃO}

Nos dias atuais, a concorrência é global e o foco dos negócios precisa estar cada vez mais voltado para o consumidor. Entender seus desejos e necessidades é vital para criação e desenvolvimento de produtos e serviços. Seguindo essa premissa, o estudo procurou demonstrar a percepção dos consumidores com relação a um serviço inovador, o Mobile Banking.

Com o teste de variância, buscou-se identificar as diferentes percepções entre adotantes e não adotantes quanto a percepção dos atributos de uma inovação. Concluiu-se que, com relação à vantagem relativa, os adotantes acreditam nas vantagens relacionadas ao custo que o serviço proporciona e na facilidade de operação (baixa complexidade).

Já os não adotantes percebem o serviço como pouco versátil e têm mais dificuldade em contar para os outros sobre o uso do MB. Outro aspecto a ser considerado é a percepção de que o ambiente do MB é menos seguro. Por isso, questões de segurança têm importância relevante quando se trata de operações bancárias e é importante que os mecanismos para prevenção de fraudes fiquem evidenciados, deixando o consumidor mais confiante para adotar.

Os resultados obtidos com a correlação dos dados mostraram haver relação entre as percepções acerca dos atributos de produtos inovadores e características de personalidade inovadora, portanto, esses resultados corroboram a maior parte dos pressupostos teóricos do modelo de difusão de inovações (TDI). A relação estabelecida na teoria foi de certa maneira a explicação para os resultados encontrados no presente estudo empírico. 0 comportamento no uso de computadores pode ser um resultado de um conjunto de crenças sobre tecnologia e um conjunto de respostas afetivas ao comportamento (Moore e Benbasat, 1991). Logo, mais pesquisas serão necessárias para que se compreenda de forma mais profunda as relações entre as características pessoais frente à inovação e 0 comportamento de adoção de determinado produto ou serviço inovador.

Podem ser constatadas limitações de tempo e recursos, por razão da aplicação dos questionários ter ficado restrita ao curso de graduação em Administração do Campus Universitário da Universidade Federal do Rio Grande do Norte, situada na região nordeste do Brasil, devido à maior facilidade e rapidez na obtenção dos resultados. No entanto, apesar de restrita, a amostra é bem qualificada, em termos demográficos e de nível de escolaridade.

Recomenda-se, para estudos futuros, o aprofundamento de pesquisas na área da adoção de tecnologias móveis, como o celular, já que o mesmo vem cada vez mais concatenando funções de outros produtos como câmeras digitais, MP3 e TV, e principalmente ao uso efetivo dessas novas possibilidades no ambiente universitário, majoritariamente jovem e propenso à adotar novas tecnologias de informação e comunicação. Outra direção é estender o estudo para incorporar os construtos do modelo de aceitação de tecnologia (TAM), bem como incluir a variável relacionada com a influência social no comportamento de adoção de inovação de tecnologia.

Do ponto de vista acadêmico, esta pesquisa buscou avançar o conhecimento do comportamento inovativo de uma população que venha a adotar serviços bancários pelo telefone celular móvel, o qual pode ser muito útil e proveitoso para as estratégias de inserção e ampliação do mobile banking no mercado brasileiro, em particular.

Quanto às implicações gerenciais, propõe-se uma atuação mais intensiva dos bancos com ações de comunicação de marketing voltadas inicialmente para os usuários de Internet Banking que ainda não são usuários do Mobile Banking. Essas ações de divulgação devem focar nas variáveis de adoção de inovações que demonstrem as vantagens relativas do uso, o baixo custo e a alta segurança das operações. Portanto, o envolvimento das agências faz-se necessário, já que essas estão em contato direto com os clientes. Propõe-se uma experimentação (trialability) do serviço nas agências de forma a 
desmistificar seu uso junto aos correntistas. Os achados desta pesquisa sinalizam que devem ser estimuladas ações de treinamento e de experimentação de produtos que caracterizam inovações tecnológicas, a fim de reduzir o medo da mudança e permitir maiores taxas de difusão do mobile banking.

\section{REFERÊNCIAS}

Anatel. Agência Brasileira de Telecomunicações. Brasil tem mais de 147 milhões de celulares. 17 de Dezembro de 2008. Acesso na Url: http://www.anatel.gov.br/Portal/exibirPortalInternet.do Anjos, A. dos. 2008. Análise de Variância. Disponível em <http://www.est.ufpr.br/ce003/material/cap7.pdf> Acesso em: 27 jun. 2008.

Arima, Kátia. 2005. Bancos investem em mobile banking. São Paulo, Jornal 0 Estado de São Paulo. Disponível em <http://www.link.estadao.com.br/index.cfm?id_conteudo=5938>. Acesso em: 23 maio 2007.

Bellini, Marcelo. 2008. Mobile Banking: o banco móvel. Folha de São Paulo. Disponível em $<$ www.folha.com.br>. Acesso em: 10 jan.2008.

Bueno, Ubiratan; Zwicker, Ronaldo; Oliveira, Mauri A. 2004. Um estudo comparativo do modelo de aceitação de tecnologia aplicado em sistemas de informação e comércio eletrônico. Congresso Internacional de Gestão de Tecnologia e Sistemas de Informação. São Paulo, Brasil, pp. 1-20.

Fachin, Odília. 2002. Fundamentos de Metodologia. São Paulo: Saraiva.

Filho, Bento A. da C.; Pires, Péricles José. 2005. Avaliação dos Fatores Relacionados na Formação do Índice de Prontidão À Tecnologia - TRI (Technology Readiness Index) como Antecedentes do Modelo TAM (Technology Acceptance Model). XXIX Encontro da Associação Nacional de Pós-Graduação e Pesquisa em Administração. Brasília, Brasil, pp. 1-15

Hernandez, J. M. C.; Mazzon, J. A. 2006. Adoção de Internet Banking: Proposição e Aplicação de Uma Abordagem Metodológica Integrada. XXX Encontro da Associação Nacional de Pós-Graduação e Pesquisa em Administração. Salvador, Brasil.

Hung, Shin-Yuan; Ku, Cheng-Yuan; Chang, Chia-Ming. 2002. Critical factors of WAP services adoption: an empirical study. Electronic Commerce Research and Applications. Vol. 2, №. 1, pp. 42-60. IBOPE/NetRatings. Disponível em: $<$ http://www.ibope.com.br/calandraWeb/servlet/CalandraRedirect?temp=0\&proj=PortalIBOPE\&pub $=\mathrm{T} \& \mathrm{db}=\mathrm{caldb}>$ Acesso em: 19 maio 2007.

Instituto Brasileiro de Geografia e Estatística (IBGE). 2005. Disponível em: <http://www.ibge.gov.br/home/estatistica/populacao/acessoainternet/defaulttab_hist_.shtm> Acesso em: 19 maio 2007.

Kalakota, Ravi; Robinson, Márcia. 2002. M-business: tecnologia móvel e estratégia de negócios. Bookman, Porto Alegre.

Löbler, Mauri L.; Visentini, Monize S.; Vieira, Kelmara M. A Aceitação do Comércio Eletrônico Explicada pelos Modelos TAM e TTF Combinados. XXX Encontro da Associação Nacional de Pós-Graduação e Pesquisa em Administração. Salvador, Brasil.

Lu, June; Yao, James E.; Yu, Chun-Sheng. 2005. Personal innovativeness, social influences and adoption of wireless Internet services via mobile technology. Journal of Strategic Information Systems. Vol. 14, p.245-268.

Luarn, Pin; Lin, Hsin-Hui. 2005. Toward an understanding of the behavioral intention to use mobile banking. Computers Human Behavior, Vol. 21, N․ 6, pp. 873-891.

Martins, Gilberto de A.; Lintz, Alexandre. 2000. Guia para elaboração de monografias e trabalhos de conclusão de curso. Atlas, São Paulo.

Moore, Gary C.; Benbasat, Izak. 1991. Development of an Instrument to Measure the Perceptions of 
Diferenças de percepção de adotantes e não-adotantes quanto ao uso de serviços de mobile banking e sua relação com as características individuais de inovatividade

Adopting an Information Technology Innovation. Information Systems Research, v.2, n.3, p.192-222.

Oliveira, Ana.P.. 2006. Bancos enfim sucumbem aos encantos do mobile banking. Computerworld. Disponível em:<http://idgnow.uol.com.br/computerworld/comunicacoes/2006/08/08/idgnoticia.200608-04.4624377473/redirectViewEdit?pageNumber:int=2> Acesso em: 15 maio.2007.

Prado, Paulo H. M.; Pádua Jr, Fábio Pimenta de. 2005. A Adoção de Inovações em Produtos de Alta Tecnologia por Jovens: 0 Caso do Telefone Celular. XXIX Encontro da Associação Nacional de PósGraduação e Pesquisa em Administração. Brasília, Brasil, pp. 534-534.

Parasuraman, A.; Colby, Charles L. 2002. Marketing para produtos inovadores: como e porque seus clientes adotam tecnologia. Bookman: Porto Alegre.

Quintella, Heitor M.; Peliccione, Fabiano. 2006. Análise dos fatores críticos de sucesso no lançamento do multi-protocol label switching (mpls) no mercado de telecomunicações para serviços aéreos brasileiros. Relatórios de Pesquisa em Engenharia de Produção. Vol. 6, №. 10.

Rodrigues, Ana Flavia B. de Bello; Marchetti, Renato Z. 2008. Fatores Explicativos do Comportamento Inovador entre Internautas na Idade Madura. XXXII Encontro da Associação Nacional de PósGraduação e Pesquisa em Administração, Rio de Janeiro, Brasil.

Rogers, Everett. 1995. Diffusion of innovations. Free Press, New York.

Saccol, Amarolinda I. da C. Z.; Reinhard, Nicolau. 2004. Tecnologias de Informação Móveis, Sem Fio e Ubíquas: Definições, Mapeamento do Estado-da-Arte e Oportunidades de Pesquisa. XXVIII Encontro da Associação Nacional de Pós-Graduação e Pesquisa em Administração, Curitiba, Brasil.

SISBACEN. Sistema de Informação do Banco Central. Disponível em: <http://www.relatoriobancario.com.br/noticias/noticias_ranking2_2006.html>. Acesso em 15 set.2007.

Stevens, Robert. et al. 2001. Planejamento de marketing: guia de processos e aplicações práticas. Pearson Education do Brasil, São Paulo.

Turban, Efraim; Mclean, Ephraim; Wetherbe, James. 2004. Tecnologia da informação para gestão. Bookman, Porto Alegre.

Wang, Yi-Shun; Lin, Hsin-Hui; Luarn, Pin. 2006. Predicting consumer intention to use mobile service. Information Systems Journal. Vol. 16, №. 2, pp. 157-179.

Yi, Mun. et al. 2006. Understanding information technology acceptance by individual professionals: Toward an integrative view. Information and Management. Vol. 43, p. 350 - 363. 